\title{
A Mean Curvature Based Primal Sketch to Study the Cortical Folding Process from Antenatal to Adult Brain
}

\author{
A. Cachia ${ }^{1,4,5}$, J.-F. Mangin ${ }^{1,5}$, D. Rivière ${ }^{1,5}$, N. Boddaert $^{2,5}$, A. Andrade ${ }^{1,5}$, \\ F. Kherif ${ }^{1,5}$, P. Sonigo ${ }^{2,5}$, D. Papadopoulos-Orfanos ${ }^{1,5}$, M. Zilbovicius ${ }^{1,5}$, \\ J.-B. Poline ${ }^{1,5}$, I. Bloch ${ }^{4,5}$, F. Brunelle ${ }^{2,5}$, and J. Régis ${ }^{3}$ \\ 1 Service Hospitalier Frédéric Joliot, CEA, 91401 Orsay, France \\ mangin@shfj.cea.fr, http://www-dsv.cea.fr/ \\ 2 Service de Radiologie Pédiatrique, Hopital Necker, Paris \\ 3 Service de Neurochirurgie Fonctionnelle et Stéréotaxique, La Timone, Marseille \\ 4 Département Traitement du Signal et des Images, CNRS URA 820, ENST, Paris \\ ${ }^{5}$ Institut Fédératif de Recherche 49
}

\begin{abstract}
In this paper, we propose a new representation of the cortical surface that may be used to study the cortex folding process and to recover foetus sulcal roots usually burried in the depth of adult brains. This representation is a primal sketch derived from a scale space computed for the mean curvature of the cortical surface. This scale-space stems from a geodesic diffusion equation conditionaly to the cortical surface. The primal sketch is made up of objects defined from mean curvature minima and saddle points. The resulting sketch aims first at highlighting significant elementary folds, second at representing the fold merging process during brain growth. The relevance of the framework is illustrated by the study of central sulcus sulcal roots in antenatal, baby and adult images.
\end{abstract}

\section{Introduction}

The most striking, interesting, yet poorly understood gross morphological features of the human cerebral cortex are the diverse and complex arrangements of gyri and sulci 22]. Cortical folding patterns exhibit various forms in different individuals [13. This prevents the brain mapping community from using them as a straightforward and accurate referential to localize functional activations. Recent works claim that the solution should stem from a better understanding of the brain growth process 14, 18. The primary cortical folds that appear on the foetus cortex, indeed, seem to be especially stable across individuals. During ulterior stages of brain growth, these sulcal roots merge with each other and form different patterns depending on the subjects. Deformations of the depth of adult cortical sulci corresponding to burried gyri, however, may be some clues to the sulcal root fusions (see Fig. 10 [14, 10]. Therefore, a map of these sulcal roots 
may turn out to be the adequate generic model required to match different adult folding patterns. Moreover, a map including statistics on sulcal roots chronology and dates of appearance may become a precious tool for early detection of development problems suspected from antenatal MR images.

In this paper, we propose a new representation of the cortical surface that may be used to study the cortex folding process and to recover sulcal roots usually buried in the depth of adult brains. This representation is a primal sketch [12 1] derived from a scale space [23, 7] computed for the mean curvature of the cortical surface. This scale-space stems from a diffusion equation geodesic to the cortical surface. The primal sketch is made up of objects defined from mean curvature extrema and saddle points, like in previous approaches [8, 9]. The resulting sketch aims first at highlighting significant elementary folds, second at representing the fold merging process during brain growth. The method has been tested with antenatal, baby and adult MR images. Antenatal and children images have been acquired for clinical purposes.

\section{Spherical Mesh of the Cortical Surface}

The first stage of the method consists in extracting a smooth mesh representing the cortical surface. This mesh is endowed with the actual spherical topology of this surface, which allows the implementation of geodesic diffusion or inflation operations. In the case of children and adult brains, T1-weighted MR images obtained from an inversion recovery sequence are used. In the case of antenatal or small baby brains, the axon myelinisation is still in progress, which means that T1-weighted images show no contrast between gray and white matters. Hence T2-weighted images, which provide a better contrast (see Fig. 1) but a larger slice thickness, are acquired.

An automatic robust method for detecting a white matter interface of spherical topology from T1-weighted images has been previously described [11]. This method has been adapted to T2-weighted images in order to yield a semiautomatic toolbox dedicated to fetus and baby brains. Designing a fully automatic method was beyond the scope of this adaptation because of the frequent artefacts induced by fetus motions. The interface currently detected to represent the cortical surface is located between the cerebro spinal fluid and the brain tissues. While this choice is sufficient to detect dimples bound to become a fold, further work should be done to detect the gray/white matter interface.

The method mentioned above provides a binary mask endowed with a spherical topology interface. A standard facet tracking algorithm is used first to compute a spherical graph made up of facets [5]. Then, the center of each facet is connected to the center of the neighboring facets in order to yield a smooth spherical mesh of triangles. This algorithm which preserves the initial topology relies on a look-up table of configurations like in the marching cube algorithm. Finally, a decimation including smoothing is performed to discard stair artefacts related to the underlying discretization. The decimation algorithm is inspired by the algorithm used in the Vtk package [20]. The embedded smoothing operation 
slightly moves nodes towards their neighborhood gravity center, which may be related to some usual surface evolution processes [15].

\section{Mean Curvature}

Different approaches can be used to study fine details of the cortical surface folding patterns. Depth maxima have been used to detect a concept similar to sulcal roots in [10]. In this paper, mean curvature $(H)$ is proposed as a richer descriptor (than the depth) of the various features that can be observed along sulcus bottoms and walls, which is illustrated in figure 2 fold bottoms appear as local minima of $H$ while gyrus crown appear as maxima. Hence, burried gyri appear as areas of positive curvature along the sulcus walls. Other curvature related features, such as Koenderink's curvature metric C (the L2 norm of the principal curvatures, or the logarithm thereof) or the maximum principal curvature, may be interesting for our purpose and will be investigated in the future. In this paper, mean curvature is directly estimated from the mesh thanks to its relative smoothness. We used an approximation proposed in 19] that takes into account some local properties of the mesh, as triangle angles and areas, dihedral angles between normals and edge lengths (see Fig. 2). This method is considered to be as accurate and robust as quadratic patch based approaches.

\section{Scale Space and Geodesic Diffusion}

The curvature map of the cortical surface contains much geometrical information that may be related to the anatomical elements that have to be detected (sulcal roots, sulci, etc). These elements, however, correspond to different levels of scale (see Fig. 31). Moreover, a scale based point of view is required to distinguish anatomical elements from noise features bound to appear in curvature approximations (see Fig. 2). The scale-space paradigm has been developed to deal with such problems where all the scales can be of interest. Among the many possible scale-space approaches that could be used to study cortex shapes, we have chosen in this paper a standard heat equation [7] applied to mean curvature. One intriguing feature of our method that will be discussed later is the fact that heat equation has been implemented geodesically to the surface from which the mean curvature map stems.

The fact that the $2 \mathrm{D}$ lattice is embedded in a volume raises a problem concerning the parameterization upon which to base the estimation of the local partial derivatives. The parameterization adopted was a simple local transformation that maps each surface element (a node and its first neighbours) into a plane, while keeping unchanged both edge distances and angular proportions between the edges [21, 2]. An individual parameterization is defined for each node. Locally mapping each surface element into the plane avoids the severe areal distortion that would result from a global flattening.

Implementation of partial differential equations on irregular lattices can lead to complex problems. The causality property usually required by the scale-space 
framework may be lost because of discrete phenomena. This point is beyond the scope of this paper and would require further study. Our implementation is carried out as an iterative process of the form:

$$
H(M, t+\delta t)=H(M, t)+\delta t \widehat{\Delta} H(M, t)
$$

for each point $M$ and each temporal iteration step $\delta t$, where $\widehat{\Delta}$ is an estimate of a Laplacian. Using the 2D parameterization, the Laplacian can be estimated using the approach proposed in [6], based on Taylor series expansion of a function around a point. For each neighbor $\left(u_{i}, v_{i}\right)$ of a given point $M=\left(u_{0}, v_{0}\right)$, this expansion has the form:

$$
H_{i}=H_{0}+h_{i} \frac{\partial H_{0}}{\partial u}+k_{i} \frac{\partial H_{0}}{\partial v}+\frac{h_{i}^{2}}{2} \frac{\partial^{2} H_{0}}{\partial u^{2}}+\frac{k_{i}^{2}}{2} \frac{\partial^{2} H_{0}}{\partial v^{2}}+k_{i} h_{i} \frac{\partial^{2} H_{0}}{\partial u \partial v}+O\left(\delta^{3}\right)
$$

$H_{i}=H\left(u_{i}, v_{i}\right), \frac{\partial H_{0}}{\partial u}=\frac{\partial H}{\partial u}\left(u_{0}, v_{0}\right), h_{i}=u_{i}-u_{0}, k_{i}=v_{i}-v_{0}$, and $\delta=\sqrt{h_{i}^{2}+k_{i}^{2}}$. Writing Equation 2 for all neighbors $i=1,2, \ldots m$, we obtain for each point $M$ the set of equations

$$
\begin{aligned}
& {[A][D H]-[H]=[0]} \\
& {[A]=\left[\begin{array}{ccccc}
h_{1} & k_{1} & \frac{h_{1}^{2}}{2} & \frac{k_{1}^{2}}{2} & h_{1} k_{1} \\
h_{2} & \ldots & \ldots & \ldots & \ldots \\
\vdots & & & & \\
\vdots & & & & \\
h_{m} & & & &
\end{array}\right]} \\
& {[H]=\left[H_{1}-H_{0}, \ldots, H_{m}-H_{0}\right]^{T},[D H]=\left[\frac{\partial H_{0}}{\partial u}, \frac{\partial H_{0}}{\partial v}, \frac{\partial^{2} H_{0}}{\partial u^{2}}, \frac{\partial^{2} H_{0}}{\partial v^{2}}, \frac{\partial H_{0}^{2}}{\partial u \partial v}\right]^{T} \text {. }}
\end{aligned}
$$

This system of equations is solved once at the beginning of the diffusion process.

The initial mesh construction includes some smoothing operations that may remove some interesting anatomical information. This smoothing process appears important to get initial acceptable mean curvature maps, but other diffusion processes could be less restrictive. Surface evolution according to a function of mean curvature could be an interesting alternative to our geodesic smoothing process [15]. This evolution, however, can not be implemented according to the powerful level set framework, because of the implicit definition of the surface. This framework, indeed, allows topological modification of the tracked isosurface, and provides no constant parameterization that may be used to track objects across scales. A mesh based implementation, in return, may be used for the evolution process. While a similar implementation is used to inflate our cortical surfaces [4, this last algorithm includes additional constraints minimizing local distorsions. Therefore, no such attempt has been done for the moment. It should be understood, however, that mean curvature should be recomputed during each iteration, which could be incompatible with the causality constraint of the scale-space framework. Finally, anisotropic diffusion scheme could lead to other interesting improvements of our framework [16]. 


\section{The Primal Sketch of the Mean Curvature}

A primal sketch is constructed from the mean curvature scale-space using the algorithm proposed by Lindeberg applied to the opposite of the mean curvature [9]. Grey level (GL) blobs are extracted first from each level of scale. One GL blob is defined from each local minimum of the smoothed mean curvature. The blob extent is defined from a water rise like algorithm which is stopped by saddle points or background. Hence, each GL blob is defined by two extremal points,maximum and stop point, whose behavior in the scale-space is well known from a theoretical point of view. These extremal points appear or disappear according to events called anihilation, merge or split. GL blobs are linked across scales in order to create scale-space blobs supposed to correspond to the objects embedded in the initial curvature map. A scale-space blob is made up of a chain of GL blobs beginning and ending by one of the events mentionned above.

\section{Results and Discussion}

Figure 4 provides a glimpse on the primal sketch focused on the central sulcus of an adult brain. The structure of this sub-sketch is consistent with our initial aims. First, the three highest scale-space blobs are linked by an event which seems to correspond to the merge of the central sulcus sulcal roots described by neuroanatomists [14]. Second, the two sulcal roots have a longer life time throughout scales than noise related blobs. A fine analysis of the lower part of the sketch, however, shows that some instabilities may stem from spurious split events induced by the elongated shape of the sulcus related blobs. This will lead us to add some pruning operation into the standard approach.

The new representation of the sulcal folding patterns presented in this paper will be used to infer a finer grained than usual generic model of the human cortical surface. While such results would greatly improve our current understanding of the cortex inter-individual matching issues, a lot of anatomical work remains to be done to find the link between foetal patterns of sulcal roots, and their primal sketch analogue. While a first manual exploration is required to match the new representations with our current sulcal root maps, an automatic strategy should be devised to get a more reliable generic model. Few approaches have been proposed for such inference of high level models of brain anatomy. Some ideas could stem from similar work done from skull crest lines [17]. Another attractive direction consists of Markovian models for the comparison of primal sketches developed to match activation maps across individuals [3].

\section{References}

[1] H. Asada and M. Brady. The curvature primal sketch. IEEE PAMI, 8:2-14, 1986.

[2] A. Andrade, F. Kherif, J.-F. Mangin, K. Worsley, A.L. Paradis, O. Simon, S. Dehaene, D. Le Bihan and J.-B. Poline Detection of fMRI activation using cortical surface mapping Human Brain Mapping, 12(2):79-93, 2001. 


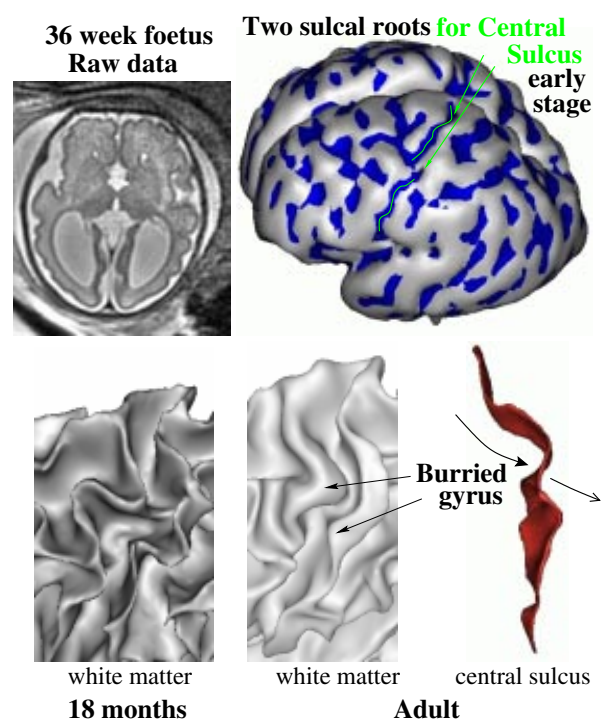

Fig. 1. Evolution of the central sulcus shape during brain growth. Up: Antenatal images allow the reconstruction of the foetus cortex surface on which shallow dimples corresponding to negative mean curvature areas are highligted in blue. At that stage, the central sulcus is made up of two sulcal roots. Down left: 18 months after birth, the gyrus separating the two sulcal roots is still visible on white matter surface. Down right: At adult stage, only slight deformations of the central sulcus walls give clues on the presence of a burried gyrus.

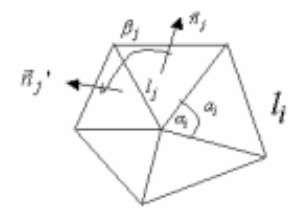

$$
H=\frac{\frac{1}{4} \sum_{j} \beta_{j} \cdot l_{j}}{\frac{1}{2} \sum_{i} a_{i}-\frac{1}{8} \sum_{i} l_{i}^{2} \cot \left(\alpha_{i}\right)}
$$

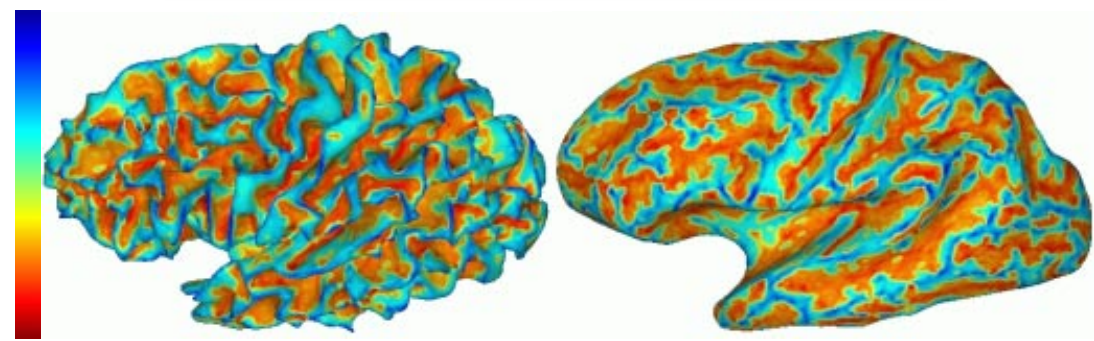

Fig. 2. Up: Approximation of mean curvature from an irregular mesh. Down: Mean curvature of the white matter surface (adult brain), mapped on itself (left) and on an inflated version [4] (right). Red (negative) areas correspond to sulci, while blue (positive) areas correspond to gyri. 


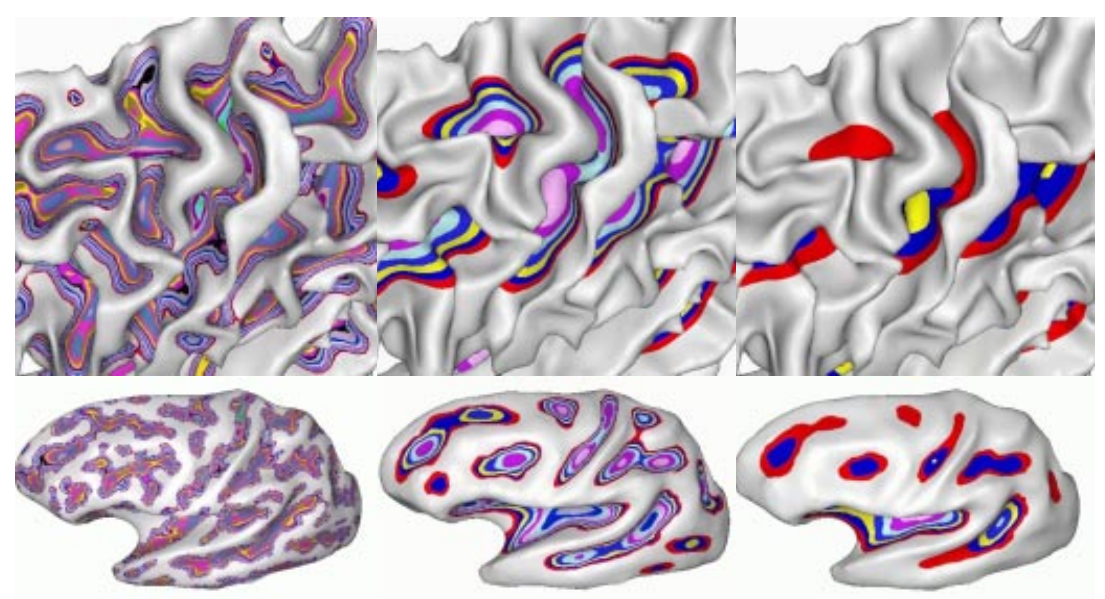

Fig. 3. Some isophotes of mean curvature at different scales. Central sulcus includes two curvature minima at middle scale, and finally only one minima at highest scale. The middle scale minima will correspond to two blobs in the final primal sketch. The saddle point which separates these two blobs is located at the level of the burried gyrus related clues. Hence, these blobs may correspond to central sulcus sulcal roots.

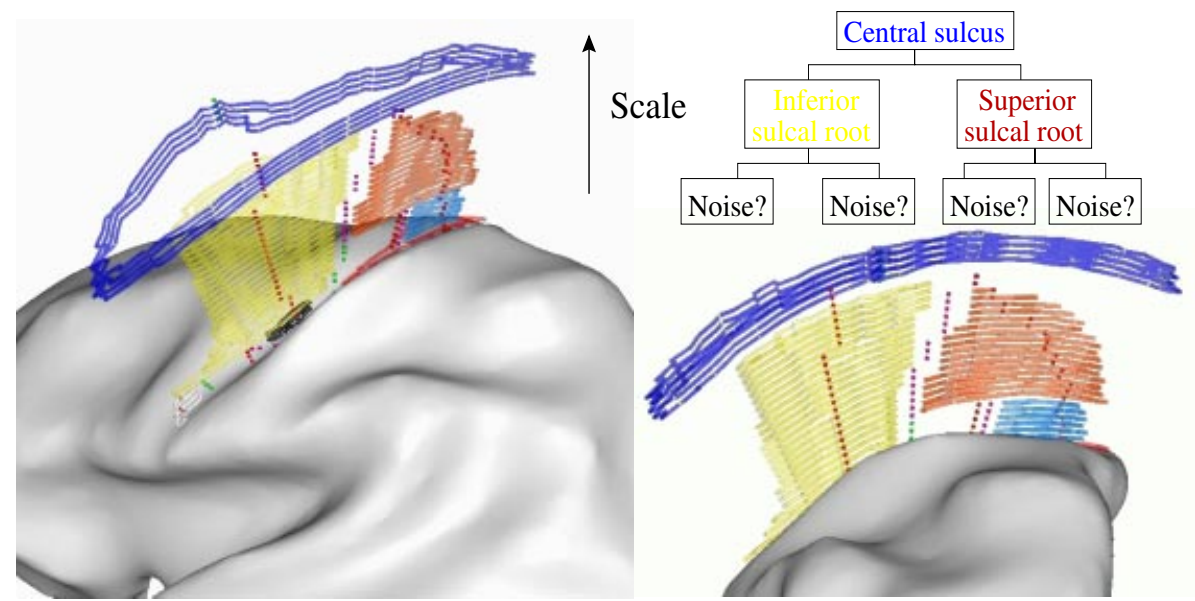

Fig. 4. Primal sketch of the mean curvature of the central sulcus. Each scalespace blob has its own color. Red points correspond to the curvature minima from which the grey level blob growth begins. Purple points between two blobs and green points between one blob and the background, correspond to points stopping blob growth. 
[3] O. Coulon, J.-F. Mangin, J.-B. Poline, M. Zilbovicius, D. Roumenov, Y. Samson, V. Frouin, and I. Bloch. Structural group analysis of functional activation maps. NeuroImage, 11:767-782, 2000.

[4] H.A. Drury, D.C. Van Essen, C. H. Anderson et al. Computerized mappings of the cerebral cortex : a multiresolution flattening method and a surface based coordinate system. Journal of cognitive neuroscience, 8(1):1-28, 1996.

[5] D. Gordon and J. Udupa. Fast surface tracking in three-dimensional binary images. Computer Vision, Graphics, and Image Processing, 45(6):196-214, 1989.

[6] G. Huiskamp. Different formulas for the surface Laplacian on a triangulated surface. Journal of computational physics, 95:477-496, 1991.

[7] J. Koenderink and A. van Doorn. The structure of images. Biological Cybernetics, 53:383-396, 1984.

[8] L. Lifshitz and S. Pizer. A multiresolution hierarchical approach to image segmentation based on intensity extrema. IEEE Trans. PAMI, 12(6):529-540, 1990.

[9] T. Lindeberg. Detecting salient blob-like image structures and their scales with a scale-space primal sketch: a method for focus-of-attention. International Journal of Computer Vision, 11(3):283-318, 1993.

[10] G. Lohmann and D. Y. von Cramon. Automatic labelling of the human cortical surface using sulcal basins. Medical Image analysis, 4(3):179-188, 2000.

[11] J.-F. Mangin, V. Frouin, I. Bloch, J. Regis, and J. López-Krahe. From 3D MR images to structural representations of the cortex topography using topology preserving deformations. J. Mathematical Imaging and Vision, 5(4):297-318, 1995.

[12] D. Marr. Vision. W. H. Freeman, NewYork, 1982.

[13] M. Ono, S. Kubik, and C. D. Abernethey. Atlas of the Cerebral Sulci. Georg Thieme Verlag, 1990.

[14] J. Régis, J.-F. Mangin, V. Frouin, F. Sastre, J. C. Peragut, and Y. Samson. Generic model for the localization of the cerebral cortex and preoperative multimodal integration in epilepsy surgery. Stereotactic Functional Neurosurgery, 65:72-80, 1995.

[15] J. A. Sethian. Level set methods. Cambridge University Press, 1996.

[16] N. Sochen, R. Kimmel, and R. Malladi. A general framework for low level vision. IEEE transactions on image processing, 20(5):100-107, 1999.

[17] G. Subsol, J.-P. Thirion, and N. Ayache. A general scheme for automatically building 3D morphometric anatomical atlases: application to a skull atlas. Medical Image Analysis, 2(1):37-60, 1998.

[18] D. C. Van Essen. A tension-based theory of morphogenesis and compact wiring in the central nervous system. Nature, 385:313-318, 1997.

[19] $\mathrm{Ph}$. Veron, D. Lesage, and J-C. Leon. Outils de base pour l'extraction de caracteristiques de surfaces numerisees. In 7eme assises europeene du prototypage rapide - Ecole Centrale de Paris, 1998.

[20] W. Schroeder,J. Zarge, and W. Lorensen Decimation of triangle meshes. In SIGGRAPH'92, pages 65-70, 1992.

[21] W. Welch and A. Witkin. Free-form shape design using triangulated surfaces. Proceedings of SIGGRAPH '94, Computer Graphics Proceedings, Annual Conference Series (July 1994, Orlando, Florida), pages 247-256, 1994.

[22] W. Welker. Why does the cerebral cortex fissure and fold. Cerebral Cortex, 8B:3$135,1989$.

[23] A. Witkin. Scale-space filtering. In Int. Joint. Conf. on Artificial Intelligence, pages 1019-1023, 1983. 